\title{
AN ALGORITHM FOR CONSTRUCTING MULTIDIMENSIONAL BIORTHOGONAL PERIODIC MULTIWAVELETS
}

\author{
SAY SONG GOH ${ }^{1}$ AND K. M. TEO ${ }^{2}$ \\ ${ }^{1}$ Department of Mathematics, National University of Singapore, \\ 10 Kent Ridge Crescent, Singapore 119260, Republic of Singapore \\ ${ }^{2}$ Hwa Chong Junior College, 661 Bukit Timah Road, \\ Singapore 269734, Republic of Singapore
}

(Received 12 November 1998)

\begin{abstract}
This paper deals with the problem of constructing multidimensional biorthogonal periodic multiwavelets from a given pair of biorthogonal periodic multiresolutions. Biorthogonal polyphase splines are introduced to reduce the problem to a matrix extension problem, and an algorithm for solving the matrix extension problem is derived. Sufficient conditions for collections of periodic multiwavelets to form a pair of biorthogonal Riesz bases of the entire function space are also obtained.
\end{abstract}

Keywords: multidimensional biorthogonal periodic multiwavelets; matrix extension

AMS 1991 Mathematics subject classification: Primary 41A63; 41A65; 42C15

\section{Introduction}

One of the important problems in wavelet analysis is the construction of biorthogonal wavelets or multiwavelets from a given pair of biorthogonal multiresolutions. Here, we consider this problem for the space $L^{2}\left([0,2 \pi)^{s}\right), s \geqslant 1$, of $2 \pi$-periodic square-integrable complex-valued functions over $[0,2 \pi)^{s}$.

For the space $L^{2}(\mathbb{R})$, it is well known that biorthogonal wavelets with dilation 2 can be constructed from multiresolutions generated by biorthogonal scaling functions with the use of simple alternating flip formulae. The problem of constructing biorthogonal multiwavelets is much more complicated. The paper by Goh and Yap [9] contains an algorithmic approach for the construction of biorthogonal multiwavelets with arbitrary dilation from multiresolutions generated by several biorthogonal scaling functions. However, such an algorithm is unavailable for $L^{2}\left(\mathbb{R}^{s}\right), s>1$, with arbitrary dilation matrices.

This paper presents such an algorithm for the space $L^{2}\left([0,2 \pi)^{s}\right), s \geqslant 1$. Periodic wavelets and multiwavelets were studied in one or more dimensions in $[1,2,4,8,11-13]$, but there is still no work done in the biorthogonal setting. To fix notation, let $M$ be an $s \times s$ matrix with integer entries such that all its eigenvalues lie outside the unit circle. 
For $k \geqslant 0$, let $\mathcal{L}_{k}$ denote a full collection of coset representatives of $\mathbb{Z}^{s} / M^{k} \mathbb{Z}^{s}$. Then

$$
\mathbb{Z}^{s}=\bigcup_{\ell \in \mathcal{L}_{k}}\left(\ell+M^{k} \mathbb{Z}^{s}\right)
$$

and, for any distinct $\ell_{1}, \ell_{2} \in \mathcal{L}_{k},\left(\ell_{1}+M^{k} \mathbb{Z}^{s}\right) \cap\left(\ell_{2}+M^{k} \mathbb{Z}^{s}\right)=\emptyset$. Similarly, we let $\mathcal{R}_{k}$ denote a full collection of coset representatives of $\mathbb{Z}^{s} / D^{k} \mathbb{Z}^{s}$, where $D=M^{\mathrm{T}}$.

For $k \geqslant 0$, consider $\mathcal{S}\left(M^{k}\right)^{r \times \rho}$, the class of periodic sequences of $r \times \rho$ complex matrices of period $M^{k}$, that is, $H_{k}\left(\ell+M^{k} p\right)=H_{k}(\ell)$ for all $H_{k} \in \mathcal{S}\left(M^{k}\right)^{r \times \rho}, \ell, p \in \mathbb{Z}^{s}$. For $H_{k} \in \mathcal{S}\left(M^{k}\right)^{r \times \rho}$, we define its finite Fourier transform by

$$
\hat{H}_{k}(j)=\sum_{\ell \in \mathcal{L}_{k}} H_{k}(\ell) \mathrm{e}^{-\mathrm{i} j \cdot\left(2 \pi M^{-k} \ell\right)}, \quad j \in \mathcal{R}_{k}
$$

Then $\hat{H}_{k} \in \mathcal{S}\left(D^{k}\right)^{r \times p}$, and the sequence $H_{k}$ can be recovered from $\hat{H}_{k}$ by

$$
H_{k}(\ell)=\frac{1}{\left|\mathcal{R}_{k}\right|} \sum_{j \in \mathcal{R}_{k}} \hat{H}_{k}(j) \mathrm{e}^{\mathrm{i} \ell \cdot\left(2 \pi D^{-k} j\right)}, \quad \ell \in \mathcal{L}_{k} .
$$

In addition,

$$
\frac{1}{\left|\mathcal{R}_{k}\right|} \sum_{j \in \mathcal{R}_{k}}\left|\hat{H}_{k}(j)\right|^{2}=\sum_{\ell \in \mathcal{L}_{k}}\left|H_{k}(\ell)\right|^{2}, \quad H_{k} \in \mathcal{S}\left(M^{k}\right)
$$

where $\mathcal{S}\left(M^{k}\right):=\mathcal{S}\left(M^{k}\right)^{1 \times 1}$.

We say that a sequence of subspaces $\left\{V_{k}: k \geqslant 0\right\}$ of $L^{2}\left([0,2 \pi)^{s}\right)$ is a periodic multiresolution of $L^{2}\left([0,2 \pi)^{s}\right)$ with multiplicity $r$ and dilation matrix $M$ if it satisfies the following conditions.

(MR1) For $k=0,1, \ldots, \operatorname{dim} V_{k}=r\left|\operatorname{det}\left(M^{k}\right)\right|$, and there exist functions $\phi_{k}^{m} \in V_{k}$, $m=1,2, \ldots, r$, such that $\left\{T_{k}^{\ell} \phi_{k}^{m}: m=1,2, \ldots, r, \ell \in \mathcal{L}_{k}\right\}$ is a basis for $V_{k}$, where $T_{k}^{\ell} f:=f\left(\cdot-2 \pi M^{-k} \ell\right)$.

(MR2) For $k=0,1, \ldots, V_{k} \subseteq V_{k+1}$.

(MR3) $\overline{\bigcup_{k \geqslant 0} V_{k}}=L^{2}\left([0,2 \pi)^{s}\right)$.

The functions $\phi_{k}^{m}, k \geqslant 0, m=1,2, \ldots, r$, are called scaling functions. Suppose that $\left\{\tilde{V}_{k}: k \geqslant 0\right\}$ is another periodic multiresolution of $L^{2}\left([0,2 \pi)^{s}\right)$ with multiplicity $r$ and dilation matrix $M$ generated by scaling functions $\tilde{\phi}_{k}^{m}, k \geqslant 0, m=1,2, \ldots, r$. The two multiresolutions $\left\{V_{k}: k \geqslant 0\right\}$ and $\left\{\tilde{V}_{k}: k \geqslant 0\right\}$ are said to be biorthogonal if the collections $\left\{T_{k}^{\ell} \phi_{k}^{m}: m=1,2, \ldots, r, \ell \in \mathcal{L}_{k}\right\}$ and $\left\{T_{k}^{\ell} \tilde{\phi}_{k}^{m}: m=1,2, \ldots, r, \ell \in \mathcal{L}_{k}\right\}$ are biorthogonal for every $k \geqslant 0$, that is,

$$
\left\langle T_{k}^{j} \phi_{k}^{m}, T_{k}^{\ell} \tilde{\phi}_{k}^{\mu}\right\rangle=\delta_{j \ell} \delta_{m \mu}, \quad k \geqslant 0, \quad j, \ell \in \mathcal{L}_{k}, \quad m, \mu=1,2, \ldots, r .
$$


The scaling functions $\phi_{k}^{m}$ and $\tilde{\phi}_{k}^{m}, k \geqslant 0, m=1,2, \ldots, r$, satisfy the periodic matrix refinement equations

$$
\phi_{k}=\sum_{\ell \in \mathcal{L}_{k+1}} H_{k+1}(\ell) T_{k+1}^{\ell} \phi_{k+1}, \quad \tilde{\phi}_{k}=\sum_{\ell \in \mathcal{L}_{k+1}} \tilde{H}_{k+1}(\ell) T_{k+1}^{\ell} \tilde{\phi}_{k+1}
$$

where $\phi_{k}:=\left(\phi_{k}^{1}, \ldots, \phi_{k}^{r}\right)^{\mathrm{T}}, \tilde{\phi}_{k}:=\left(\tilde{\phi}_{k}^{1}, \ldots, \tilde{\phi}_{k}^{r}\right)^{\mathrm{T}}$, and $H_{k+1}, \tilde{H}_{k+1} \in \mathcal{S}\left(M^{k+1}\right)^{r \times r}$, for every $k \geqslant 0$. Writing in terms of Fourier coefficients, (1.3) is equivalent to

$$
\hat{\phi}_{k}(n)=\hat{H}_{k+1}(n) \hat{\phi}_{k+1}(n), \quad \hat{\tilde{\phi}}_{k}(n)=\hat{\tilde{H}}_{k+1}(n) \hat{\tilde{\phi}}_{k+1}(n)
$$

for all $n \in \mathbb{Z}^{s}$.

In this paper, we seek functions $\psi_{k}^{m}, \tilde{\psi}_{k}^{m} \in L^{2}\left([0,2 \pi)^{s}\right), k \geqslant 0, m=1,2, \ldots, r(d-1)$, where $d=|\operatorname{det}(M)|$, called multiwavelets, satisfying the biorthogonality relation

$$
\left\langle T_{k}^{j} \psi_{k}^{m}, T_{k}^{\ell} \tilde{\psi}_{k}^{\mu}\right\rangle=\delta_{j \ell} \delta_{m \mu}
$$

for $k \geqslant 0, m, \mu=1,2, \ldots, r(d-1), j, \ell \in \mathcal{L}_{k}$, with the sets

$$
W_{k}:=\left\langle\left\{T_{k}^{\ell} \psi_{k}^{m}: m=1,2, \ldots, r(d-1), \ell \in \mathcal{L}_{k}\right\}\right\rangle
$$

and

$$
\tilde{W}_{k}:=\left\langle\left\{T_{k}^{\ell} \tilde{\psi}_{k}^{m}: m=1,2, \ldots, r(d-1), \ell \in \mathcal{L}_{k}\right\}\right\rangle
$$

satisfying the direct sums

$$
V_{k+1}=V_{k} \oplus W_{k}, \quad \tilde{V}_{k+1}=\tilde{V}_{k} \oplus \tilde{W}_{k},
$$

and the orthogonality properties

$$
W_{k} \perp \tilde{V}_{k}, \quad \tilde{W}_{k} \perp V_{k}
$$

for $k \geqslant 0$. Since $\operatorname{dim} V_{k}=r d^{k},(1.7)$ will imply that each of the collections $\left\{T_{k}^{\ell} \psi_{k}^{m}\right.$ : $\left.m=1,2, \ldots, r(d-1), \ell \in \mathcal{L}_{k}\right\}$ and $\left\{T_{k}^{\ell} \tilde{\psi}_{k}^{m}: m=1,2, \ldots, r(d-1), \ell \in \mathcal{L}_{k}\right\}$ is linearly independent.

In [8], a polyphase spline approach was proposed for the study of multiresolutions and multiwavelets in $L^{2}\left([0,2 \pi)^{s}\right)$ for the orthonormal and semi-orthogonal settings. Here, we extend it to the biorthogonal setting. In $\S 2$, we characterize the biorthogonality relation (1.2) in terms of polyphase splines. In $\S 3$, this characterization is used to reduce the biorthogonal multiwavelet construction problem to a matrix extension problem, and a constructive solution is provided. The paper concludes in $\S 4$ with sufficient conditions for collections of biorthogonal multiwavelets to form a pair of biorthogonal Riesz bases of $L^{2}\left([0,2 \pi)^{s}\right)$. 


\section{Characterization of biorthogonality}

For $k \geqslant 0$, let $\phi_{k}^{m}, \tilde{\phi}_{k}^{m}, m=1,2, \ldots, r$, be functions in $L^{2}\left([0,2 \pi)^{s}\right)$. Consider the sets $S_{k}:=\left\{T_{k}^{\ell} \phi_{k}^{m}: m=1,2, \ldots, r, \ell \in \mathcal{L}_{k}\right\}$ and $\tilde{S}_{k}:=\left\{T_{k}^{\ell} \tilde{\phi}_{k}^{m}: m=1,2, \ldots, r, \ell \in \mathcal{L}_{k}\right\}$. Define polyphase splines $v_{k, j}^{m}$ in $L^{2}\left([0,2 \pi)^{s}\right)$ by

$$
v_{k, j}^{m}(x):=\sum_{p \in \mathbb{Z}^{s}} \hat{\phi}_{k}^{m}\left(j+D^{k} p\right) \mathrm{e}^{\mathrm{i}\left(j+D^{k} p\right) \cdot x},
$$

for $m=1,2, \ldots, r, j \in \mathcal{R}_{k}$, where the $\hat{\phi}_{k}^{m}(n)$ denote the Fourier coefficients of $\phi_{k}^{m}$. Similarly, we define $\tilde{v}_{k, j}^{m}$ using $\tilde{\phi}_{k}^{m}(n)$, the Fourier coefficients of $\tilde{\phi}_{k}^{m}$.

The polyphase splines $v_{k, j}^{m}$ were first introduced in [8], and they were used to characterize the linear independence of the set $S_{k}$. Here, we shall use the two collections of polyphase splines $\left\{v_{k, j}^{m}: j \in \mathcal{R}_{k}, m=1,2, \ldots, r\right\}$ and $\left\{\tilde{v}_{k, j}^{m}: j \in \mathcal{R}_{k}, m=1,2, \ldots, r\right\}$ to characterize the biorthogonality of $S_{k}$ and $\tilde{S}_{k}$.

Theorem 2.1. For $k \geqslant 0$, the sets $S_{k}$ and $\tilde{S}_{k}$ are biorthogonal, that is,

$$
\left\langle T_{k}^{p} \phi_{k}^{m}, T_{k}^{q} \tilde{\phi}_{k}^{\mu}\right\rangle=\delta_{p q} \delta_{m \mu}, \quad p, q \in \mathcal{L}_{k}, \quad m, \mu=1,2, \ldots, r,
$$

if and only if

$$
\left(\left\langle v_{k, j}^{m}, \tilde{v}_{k, j}^{\mu}\right\rangle\right)_{m, \mu=1}^{r}=\left(1 / d^{k}\right) I_{r}, \quad j \in \mathcal{R}_{k},
$$

where $I_{r}$ denotes the $r \times r$ identity matrix.

Proof. First, we note that it suffices to establish the theorem for any chosen collections of coset representatives $\mathcal{L}_{k}$ and $\mathcal{R}_{k}$. Thus, we shall select the particular collections of $\mathcal{L}_{k}$ and $\mathcal{R}_{k}$ described in $[\mathbf{8}, \S 3]$. The procedure is as follows. Choose bases $\left\{e_{1}^{\prime}, \ldots, e_{s}^{\prime}\right\}$ and $\left\{e_{1}^{\prime \prime}, \ldots, e_{s}^{\prime \prime}\right\}$ of the free abelian group $\mathbb{Z}^{s}$, and sets of generators $\left\{f_{1}^{\prime}, \ldots, f_{s}^{\prime}\right\}$ and $\left\{g_{1}^{\prime}, \ldots, g_{s}^{\prime}\right\}$ of $M^{k} \mathbb{Z}^{s}$ and $D^{k} \mathbb{Z}^{s}$, respectively, such that

$$
f_{i}^{\prime}=n_{i} e_{i}^{\prime}, \quad g_{i}^{\prime}=n_{i} e_{i}^{\prime \prime}, \quad i=1,2, \ldots, s .
$$

Here, $n_{1}, \ldots, n_{s}$ are positive integers such that

$$
d^{k}=\left|\operatorname{det}\left(M^{k}\right)\right|=\left|\operatorname{det}\left(D^{k}\right)\right|=n_{1} n_{2} \cdots n_{s} .
$$

Thus, if we set

$$
\mathcal{L}_{k}=\left\{m_{1} e_{1}^{\prime}+\cdots+m_{s} e_{s}^{\prime}: 0 \leqslant m_{i}<n_{i}, i=1,2, \ldots, s\right\}
$$

and

$$
\mathcal{R}_{k}=\left\{\mu_{1} e_{1}^{\prime \prime}+\cdots+\mu_{s} e_{s}^{\prime \prime}: 0 \leqslant \mu_{i}<n_{i}, i=1,2, \ldots, s\right\},
$$

then $\mathcal{L}_{k}$ and $\mathcal{R}_{k}$ are sets of all coset representatives of $\mathbb{Z}^{s} / M^{k} \mathbb{Z}^{s}$ and $\mathbb{Z}^{s} / D^{k} \mathbb{Z}^{s}$, respectively. With this particular choice of $\mathcal{L}_{k}$, we can order $\mathcal{L}_{k}$ as in [8], and let us denote $\mathcal{L}_{k}=\left\{\ell_{1}, \ldots, \ell_{d^{k}}\right\}$. 
For each $m, \mu=1,2, \ldots, r$, consider the $d^{k} \times d^{k}$ matrix

$$
\Phi_{m \mu}:=\left(\left\langle T_{k}^{\ell_{p}} \phi_{k}^{m}, T_{k}^{\ell_{q}} \tilde{\phi}_{k}^{\mu}\right\rangle\right)_{p, q=1}^{d^{k}}=\left(\left\langle\phi_{k}^{m}, T_{k}^{\ell_{q}-\ell_{p}} \tilde{\phi}_{k}^{\mu}\right\rangle\right)_{p, q=1}^{d^{k}},
$$

which is a circulant of level $s$ and type $\left(n_{1}, \ldots, n_{s}\right)$. By [7, Theorem 5.8.4],

$$
\Phi_{m \mu}=F^{*}\left(\sum_{\gamma_{1}=0}^{n_{1}-1} \cdots \sum_{\gamma_{s}=0}^{n_{s}-1}\left\langle\phi_{k}^{m}, \tilde{\phi}_{k}^{\mu}\left(\cdot-2 \pi M^{-k}\left(\gamma_{1} e_{1}^{\prime}+\cdots+\gamma_{s} e_{s}^{\prime}\right)\right)\right\rangle \Omega^{\gamma}\right) F
$$

Here, $F=F_{n_{1}} \otimes \cdots \otimes F_{n_{s}}$ is the Kronecker product of the Fourier matrices $F_{n_{\nu}}, \nu=$ $1,2, \ldots, s$ (see [7] for the definitions), and $\Omega^{\gamma}=\Omega_{n_{1}}^{\gamma_{1}} \otimes \cdots \otimes \Omega_{n_{s}}^{\gamma_{s}}$, where

$$
\Omega_{n_{\nu}}=\operatorname{diag}\left(1, \omega_{n_{\nu}}, \omega_{n_{\nu}}^{2}, \ldots, \omega_{n_{\nu}}^{n_{\nu}-1}\right), \quad \omega_{n_{\nu}}=\exp \left(2 \pi \mathrm{i} / n_{\nu}\right) .
$$

As in $[8, \S 3]$, we deduce that the eigenvalues of $\Phi_{m \mu}$ can be written as

$$
\lambda_{m \mu}^{j}:=d^{k} \sum_{p \in \mathbb{Z}^{s}} \hat{\phi}_{k}^{m}\left(j+D^{k} p\right) \overline{\hat{\hat{\phi}}_{k}^{\mu}\left(j+D^{k} p\right)}, \quad j \in \mathcal{R}_{k}
$$

On the other hand, since

$$
\left\langle v_{k, j}^{m}, \tilde{v}_{k, j}^{\mu}\right\rangle=\sum_{p \in \mathbf{Z}^{s}} \hat{\phi}_{k}^{m}\left(j+D^{k} p\right) \overline{\hat{\tilde{\phi}}_{k}^{\mu}\left(j+D^{k} p\right)}, \quad j \in \mathcal{R}_{k},
$$

we have

$$
\lambda_{m \mu}^{j}=d^{k}\left\langle v_{k, j}^{m}, \tilde{v}_{k, j}^{\mu}\right\rangle, \quad j \in \mathcal{R}_{k}
$$

Now, if $m \neq \mu$, then $\left\langle T_{k}^{p} \phi_{k}^{m}, T_{k}^{q} \tilde{\phi}_{k}^{\mu}\right\rangle=0$ for all $p, q \in \mathcal{L}_{k}$ amounts to $\Phi_{m \mu}=0$; and it follows from (2.1) that $\Phi_{m \mu}=0$ if and only if $\lambda_{m \mu}^{j}=0$ for all $j \in \mathcal{R}_{k}$. By (2.2), this is equivalent to $\left\langle v_{k, j}^{m}, \tilde{v}_{k, j}^{\mu}\right\rangle=0$ for all $j \in \mathcal{R}_{k}$.

If $m=\mu$, then (2.1) implies that

$$
\Phi_{m m}=\left(\left\langle T_{k}^{\ell_{p}} \phi_{k}^{m}, T_{k}^{\ell_{q}} \tilde{\phi}_{k}^{m}\right\rangle\right)_{p, q=1}^{d^{k}}=I_{d^{k}}
$$

if and only if $\lambda_{m m}^{j}=1$ for all $j \in \mathcal{R}_{k}$ (since $F$ is unitary). By (2.2), this is equivalent to

$$
\left\langle v_{k, j}^{m}, \tilde{v}_{k, j}^{m}\right\rangle=\left(1 / d^{k}\right)
$$

which completes the proof of the theorem.

\section{Algorithm for multiwavelet construction}

Let $\left\{\phi_{k}^{m}: k \geqslant 0, m=1,2, \ldots, r\right\}$ and $\left\{\tilde{\phi}_{k}^{m}: k \geqslant 0, m=1,2, \ldots, r\right\}$ be two sets of scaling functions that generate a pair of biorthogonal multiresolutions $\left\{V_{k}: k \geqslant 0\right\}$ and $\left\{\tilde{V}_{k}\right.$ : $k \geqslant 0\}$ of $L^{2}\left([0,2 \pi)^{s}\right)$ with multiplicity $r$ and dilation matrix $M$. We shall find functions $\psi_{k}^{m}, \tilde{\psi}_{k}^{m} \in L^{2}\left([0,2 \pi)^{s}\right), k \geqslant 0, m=1,2, \ldots, r(d-1)$, satisfying the biorthogonality 
relation (1.4), with the sets $W_{k}$ and $\tilde{W}_{k}, k \geqslant 0$, in (1.5) and (1.6) satisfying (1.7) and (1.8). Our strategy is to construct appropriate polyphase splines in $L^{2}\left([0,2 \pi)^{s}\right)$ of the form

$$
\begin{aligned}
& u_{k, j}^{m}(x):=\sum_{p \in \mathbb{Z}^{s}} b_{k}^{m}\left(j+D^{k} p\right) \mathrm{e}^{\mathrm{i}\left(j+D^{k} p\right) \cdot x} \\
& \tilde{u}_{k, j}^{m}(x):=\sum_{p \in \mathbb{Z}^{s}} \tilde{b}_{k}^{m}\left(j+D^{k} p\right) \mathrm{e}^{\mathrm{i}\left(j+D^{k} p\right) \cdot x}
\end{aligned}
$$

for $m=1,2, \ldots, r(d-1), j \in \mathcal{R}_{k}$, and then define

$$
\psi_{k}^{m}:=\sum_{j \in \mathcal{R}_{k}} u_{k, j}^{m}, \quad \tilde{\psi}_{k}^{m}:=\sum_{j \in \mathcal{R}_{k}} \tilde{u}_{k, j}^{m},
$$

for $m=1,2, \ldots, r(d-1)$. Here, $b_{k}^{m}, \tilde{b}_{k}^{m} \in \ell^{2}\left(\mathbb{Z}^{s}\right)$ are sequences such that

$$
\begin{aligned}
& \left(b_{k}^{1}(n), \ldots, b_{k}^{r(d-1)}(n)\right)^{\mathrm{T}}=\hat{G}_{k+1}(n)\left(\hat{\phi}_{k+1}^{1}(n), \ldots, \hat{\phi}_{k+1}^{r}(n)\right)^{\mathrm{T}}, \\
& \left(\tilde{b}_{k}^{1}(n), \ldots, \tilde{b}_{k}^{r(d-1)}(n)\right)^{\mathrm{T}}=\hat{\tilde{G}}_{k+1}(n)\left(\hat{\tilde{\phi}}_{k+1}^{1}(n), \ldots, \hat{\tilde{\phi}}_{k+1}^{r}(n)\right)^{\mathrm{T}}, \quad n \in \mathbb{Z}^{s},
\end{aligned}
$$

for some $\hat{G}_{k+1}, \hat{\tilde{G}}_{k+1} \in \mathcal{S}\left(D^{k+1}\right)^{r(d-1) \times r}$. This is equivalent to

$$
\psi_{k}=\sum_{\ell \in \mathcal{L}_{k+1}} G_{k+1}(\ell) T_{k+1}^{\ell} \phi_{k+1}, \quad \tilde{\psi}_{k}=\sum_{\ell \in \mathcal{L}_{k+1}} \tilde{G}_{k+1}(\ell) T_{k+1}^{\ell} \tilde{\phi}_{k+1},
$$

where $\psi_{k}:=\left(\psi_{k}^{1}, \ldots, \psi_{k}^{r(d-1)}\right)^{\mathrm{T}}, \tilde{\psi}_{k}:=\left(\tilde{\psi}_{k}^{1}, \ldots, \tilde{\psi}_{k}^{r(d-1)}\right)^{\mathrm{T}}$. Note that the sets $W_{k}$ and $\tilde{W}_{k}$ in (1.5) and (1.6) can be expressed as

$$
W_{k}=\left\langle\left\{u_{k, j}^{m}: m=1,2, \ldots, r(d-1), j \in \mathcal{R}_{k}\right\}\right\rangle
$$

and

$$
\tilde{W}_{k}=\left\langle\left\{\tilde{u}_{k, j}^{m}: m=1,2, \ldots, r(d-1), j \in \mathcal{R}_{k}\right\}\right\rangle
$$

(See [8] for details on the above general approach of constructing multiwavelets via polyphase splines.)

The desired polyphase splines, and, hence, multiwavelets, will be obtained by constructing matrices $\hat{G}_{k+1}, \hat{\tilde{G}}_{k+1} \in \mathcal{S}\left(D^{k+1}\right)^{r(d-1) \times r}$ so that (1.4), (1.7) and (1.8) are satisfied. For each $k \geqslant 0, j \in \mathcal{R}_{k}$, we define the following matrices:

$$
\begin{aligned}
M_{k}(j):=\left(\left\langle v_{k, j}^{m}, v_{k, j}^{\mu}\right\rangle\right)_{m, \mu=1}^{r}, & \tilde{M}_{k}(j):=\left(\left\langle\tilde{v}_{k, j}^{m}, \tilde{v}_{k, j}^{\mu}\right\rangle\right)_{m, \mu=1}^{r}, \\
N_{k}(j):=\left(\left\langle u_{k, j}^{m}, u_{k, j}^{\mu}\right\rangle\right)_{m, \mu=1}^{r(d-1)}, & \tilde{N}_{k}(j):=\left(\left\langle\tilde{u}_{k, j}^{m}, \tilde{u}_{k, j}^{\mu}\right\rangle\right)_{m, \mu=1}^{r(d-1)} \\
\mathcal{M}_{k}(j):=\left(\left\langle v_{k, j}^{m}, \tilde{v}_{k, j}^{\mu}\right\rangle\right)_{m, \mu=1}^{r}, & \mathcal{N}_{k}(j):=\left(\left\langle u_{k, j}^{m}, \tilde{u}_{k, j}^{\mu}\right\rangle\right)_{m, \mu=1}^{r(d-1)}
\end{aligned}
$$


By the arguments in [8], for $j \in \mathcal{R}_{k}$, we have

$$
\begin{aligned}
& M_{k}(j)=\sum_{\ell \in \mathcal{R}_{1}} \hat{H}_{k+1}\left(j+D^{k} \ell\right) M_{k+1}\left(j+D^{k} \ell\right) \hat{H}_{k+1}\left(j+D^{k} \ell\right)^{*} \\
& N_{k}(j)=\sum_{\ell \in \mathcal{R}_{1}} \hat{G}_{k+1}\left(j+D^{k} \ell\right) M_{k+1}\left(j+D^{k} \ell\right) \hat{G}_{k+1}\left(j+D^{k} \ell\right)^{*}
\end{aligned}
$$

as well as the corresponding identities for $\tilde{M}_{k}(j)$ and $\tilde{N}_{k}(j)$. Since $\left\{V_{k}: k \geqslant 0\right\}$ and $\left\{\tilde{V}_{k}: k \geqslant 0\right\}$ satisfy (MR1), by [8, Propositions 3.1 and 3.2$]$, we obtain

$$
\operatorname{det}\left(M_{k}(j)\right)>0, \quad \operatorname{det}\left(\tilde{M}_{k}(j)\right)>0, \quad k \geqslant 0, \quad j \in \mathcal{R}_{k} .
$$

Analogous to (3.1) and (3.2), we can derive the following identities:

$$
\begin{aligned}
\mathcal{M}_{k}(j) & =\sum_{\ell \in \mathcal{R}_{1}} \hat{H}_{k+1}\left(j+D^{k} \ell\right) \mathcal{M}_{k+1}\left(j+D^{k} \ell\right) \hat{\tilde{H}}_{k+1}\left(j+D^{k} \ell\right)^{*} \\
\mathcal{N}_{k}(j) & =\sum_{\ell \in \mathcal{R}_{1}} \hat{G}_{k+1}\left(j+D^{k} \ell\right) \mathcal{M}_{k+1}\left(j+D^{k} \ell\right) \hat{\tilde{G}}_{k+1}\left(j+D^{k} \ell\right)^{*}
\end{aligned}
$$

By Theorem 2.1, (1.2) is equivalent to

$$
\mathcal{M}_{k}(j)=\left(1 / d^{k}\right) I_{r}, \quad k \geqslant 0, \quad j \in \mathcal{R}_{k}
$$

Therefore, (3.4) implies that

$$
\sum_{\ell \in \mathcal{R}_{1}} \hat{H}_{k+1}\left(j+D^{k} \ell\right) \hat{\tilde{H}}_{k+1}\left(j+D^{k} \ell\right)^{*}=d I_{r} .
$$

By Theorem 2.1 again, we see that (1.4) is equivalent to

$$
\mathcal{N}_{k}(j)=\left(1 / d^{k}\right) I_{r(d-1)}, \quad k \geqslant 0, \quad j \in \mathcal{R}_{k},
$$

which, by (3.5) and (3.6), amounts to

$$
\sum_{\ell \in \mathcal{R}_{1}} \hat{G}_{k+1}\left(j+D^{k} \ell\right) \hat{\tilde{G}}_{k+1}\left(j+D^{k} \ell\right)^{*}=d I_{r(d-1)}
$$

As for the direct sums in (1.7), note that it suffices to establish the linear independence of the sets

$$
\left\{v_{k, j}^{m}, u_{k, j}^{\mu}: m=1,2, \ldots, r, \mu=1,2, \ldots, r(d-1), j \in \mathcal{R}_{k}\right\}
$$

and

$$
\left\{\tilde{v}_{k, j}^{m}, \tilde{u}_{k, j}^{\mu}: m=1,2, \ldots, r, \mu=1,2, \ldots, r(d-1), j \in \mathcal{R}_{k}\right\}
$$




\section{Writing}

$$
P_{k}(j):=\left(\left\langle v_{k, j}^{m}, u_{k, j}^{\mu}\right\rangle\right)_{m=1, \mu=1}^{r, r(d-1)}, \quad \tilde{P}_{k}(j):=\left(\left\langle\tilde{v}_{k, j}^{m}, \tilde{u}_{k, j}^{\mu}\right\rangle\right)_{m=1, \mu=1}^{r, r(d-1)}, \quad k \geqslant 0, \quad j \in \mathcal{R}_{k},
$$

we claim that the desired linear independence is equivalent to

$$
\operatorname{det}\left(\begin{array}{c|c}
M_{k}(j) & P_{k}(j) \\
\hline P_{k}(j)^{*} & N_{k}(j)
\end{array}\right)>0, \quad \operatorname{det}\left(\begin{array}{c|c}
\tilde{M}_{k}(j) & \tilde{P}_{k}(j) \\
\hline \tilde{P}_{k}(j)^{*} & \tilde{N}_{k}(j)
\end{array}\right)>0, \quad k \geqslant 0, \quad j \in \mathcal{R}_{k} .
$$

Indeed, observe that

$$
\left(\begin{array}{c|c}
M_{k}(j) & P_{k}(j) \\
\hline P_{k}(j)^{*} & N_{k}(j)
\end{array}\right)
$$

isjust the Gram matrix of $\mathcal{B}_{j}:=\left\{v_{k, j}^{m}, u_{k, j}^{\mu}: m=1,2, \ldots, r, \mu=1,2, \ldots, r(d-1)\right\}$. Hence, (3.10) implies that $\mathcal{B}_{j}$ is linearly independent for each fixed $j \in \mathcal{R}_{k}$. As

$$
\left\langle v_{k, j}^{m}, u_{k, \ell}^{\mu}\right\rangle=\left\langle v_{k, j}^{m}, v_{k, \ell}^{m^{\prime}}\right\rangle=\left\langle u_{k, j}^{\mu}, u_{k, \ell}^{\mu^{\prime}}\right\rangle=0
$$

for all $m, m^{\prime}=1,2, \ldots, r, \mu, \mu^{\prime}=1,2, \ldots, r(d-1)$ if $j \neq \ell$, we see that $\mathcal{B}_{j}$ is orthogonal to $\mathcal{B}_{\ell}$ if $j \neq \ell, j, \ell \in \mathcal{R}_{k}$. It follows that

$$
\bigcup_{j \in \mathcal{R}_{k}} \mathcal{B}_{j}=\left\{v_{k, j}^{m}, u_{k, j}^{\mu}: m=1,2, \ldots, r, \mu=1,2, \ldots, r(d-1), j \in \mathcal{R}_{k}\right\}
$$

is a linearly independent set. Similarly, we obtain the result for the other set of polyphase splines.

As a consequence of the linear independence condition characterized by (3.10), each of the collections $\left\{u_{k, j}^{m}: m=1,2, \ldots, r(d-1), j \in \mathcal{R}_{k}\right\}$ and $\left\{\tilde{u}_{k, j}^{m}: m=1,2, \ldots, r(d-\right.$ 1), $\left.j \in \mathcal{R}_{k}\right\}$ is linearly independent. In this case, the orthogonality of $W_{k}$ and $\tilde{V}_{k}$ translates into

$$
\left\langle u_{k, j}^{m}, \tilde{v}_{k, \ell}^{\mu}\right\rangle=0, \quad m=1,2, \ldots, r(d-1), \quad \mu=1,2, \ldots, r, \quad j, \ell \in \mathcal{R}_{k},
$$

which is equivalent to

$$
\left\langle u_{k, j}^{m}, \tilde{v}_{k, j}^{\mu}\right\rangle=0, \quad m=1,2, \ldots, r(d-1), \quad \mu=1,2, \ldots, r, \quad j \in \mathcal{R}_{k} .
$$

Proceeding as in [8], we deduce that (3.11) can be written as

$$
\sum_{\ell \in \mathcal{R}_{1}} \hat{G}_{k+1}\left(j+D^{k} \ell\right) \mathcal{M}_{k+1}\left(j+D^{k} \ell\right) \hat{\tilde{H}}_{k+1}\left(j+D^{k} \ell\right)^{*}=0_{r(d-1) \times r}
$$

which, by (3.6), is equivalent to

$$
\sum_{\ell \in \mathcal{R}_{1}} \hat{G}_{k+1}\left(j+D^{k} \ell\right) \hat{\tilde{H}}_{k+1}\left(j+D^{k} \ell\right)^{*}=0_{r(d-1) \times r}
$$


Similarly, $\tilde{W}_{k}$ being orthogonal to $V_{k}$ amounts to

$$
\sum_{\ell \in \mathcal{R}_{1}} \hat{\tilde{G}}_{k+1}\left(j+D^{k} \ell\right) \hat{H}_{k+1}\left(j+D^{k} \ell\right)^{*}=0_{r(d-1) \times r}
$$

Hence, the multiwavelet construction problem can now be formulated as a problem of constructing matrices $\hat{G}_{k+1}, \hat{\tilde{G}}_{k+1} \in \mathcal{S}\left(D^{k+1}\right)^{r(d-1) \times r}$ satisfying (3.8), (3.10), (3.12) and (3.13), under the assumptions of (3.1), (3.3) and (3.7). Our main theorem, Theorem 3.1, shows that such matrices can always be constructed.

Theorem 3.1. Suppose that $\phi_{k}^{m}, \tilde{\phi}_{k}^{m} \in L^{2}\left([0,2 \pi)^{s}\right), k \geqslant 0, m=1,2, \ldots, r$, generate a pair of biorthogonal multiresolutions of $L^{2}\left([0,2 \pi)^{s}\right)$ with multiplicity $r$ and dilation matrix $M$, so that (3.1), (3.3) and (3.7) hold. Then there exist

$$
\hat{G}_{k+1}, \hat{\tilde{G}}_{k+1} \in \mathcal{S}\left(D^{k+1}\right)^{r(d-1) \times r}
$$

such that (3.8), (3.10), (3.12) and (3.13) are satisfied.

Before we prove the theorem, let us establish a useful technical lemma.

Lemma 3.2. Suppose that $A$ and $\tilde{A}$ are $r \times n$ complex matrices, where $r<n$, satisfying

$$
A \tilde{A}^{*}=I_{r}
$$

Then there exist $n \times n$ complex matrices $C$ and $\tilde{C}$ of the form

$$
C=\left(\frac{A}{B}\right), \quad \tilde{C}=\left(\frac{\tilde{A}}{\tilde{B}}\right)
$$

for some $(n-r) \times n$ complex matrices $B$ and $\tilde{B}$ such that

$$
C \tilde{C}^{*}=I_{n}
$$

Proof. Let $v_{1}, \ldots, v_{r}$ be the rows of $A$. Since $A \tilde{A}^{*}=I_{r}$, it follows that the rank of $A$ is $r$. Thus, there exist $(n-r)$ linearly independent vectors $\tilde{w}_{1}, \ldots, \tilde{w}_{n-r}$ that form a basis of the orthogonal complement of the linear span of $\left\{v_{1}, \ldots, v_{r}\right\}$ in $\mathbb{C}^{n}$. Define an $n \times n$ complex matrix $\tilde{C}$ by

$$
\tilde{C}=\left(\frac{\tilde{A}}{\tilde{B}}\right)
$$

where $\tilde{B}$ is the $(n-r) \times n$ matrix formed by the vectors $\tilde{w}_{1}, \ldots, \tilde{w}_{n-r}$.

We claim that the matrix $\tilde{C}$ is invertible. Indeed, if

$$
\alpha_{1} \tilde{v}_{1}+\cdots+\alpha_{r} \tilde{v}_{r}+\beta_{1} \tilde{w}_{1}+\cdots+\beta_{n-r} \tilde{w}_{n-r}=0_{1 \times n}
$$

for some $\alpha_{1}, \ldots, \alpha_{r}, \beta_{1}, \ldots, \beta_{n-r} \in \mathbb{C}$, then, taking inner products with $v_{1}, \ldots, v_{r}$, respectively, yields $\alpha_{1}=\cdots=\alpha_{r}=0$. Since $\left\{\tilde{w}_{1}, \ldots, \tilde{w}_{n-r}\right\}$ is linearly independent, it follows that $\beta_{1}=\cdots=\beta_{n-r}=0$. Thus, $\left\{\tilde{v}_{1}, \ldots, \tilde{v}_{r}, \tilde{w}_{1}, \ldots, \tilde{w}_{n-r}\right\}$ is linearly independent. 
Now, set $C_{0}:=\left(\tilde{C}^{*}\right)^{-1}$. Writing $C_{0}=\left(A_{0} / B\right)$, where $A_{0}$ and $B$ are $r \times n$ and $(n-r) \times n$ matrices, respectively, the relation $C_{0} \tilde{C}^{*}=I_{n}$ implies that

$$
B \tilde{A}^{*}=0_{(n-r) \times r}, \quad B \tilde{B}^{*}=I_{n-r}
$$

Define $C:=(A / B)$. By (3.14), (3.15) and the fact that the rows of $\tilde{B}$ form a basis of the orthogonal complement of the linear span of the rows of $A$ in $\mathbb{C}^{n}$, we have $C \tilde{C}^{*}=I_{n}$. (This also shows that $C=\left(\tilde{C}^{*}\right)^{-1}=C_{0}$.) Hence, the proof of the lemma is complete.

Proof of Theorem 3.1. Fix $k \geqslant 0, j \in \mathcal{R}_{k}$, and let $\ell_{1}, \ell_{2}, \ldots, \ell_{d}$ denote all the elements of $\mathcal{R}_{1}$. Set

$$
\left.\begin{array}{l}
A_{k}(j):=(1 / \sqrt{ } d)\left(\hat{H}_{k+1}\left(j+D^{k} \ell_{1}\right)|\cdots| \hat{H}_{k+1}\left(j+D^{k} \ell_{d}\right)\right) \\
\tilde{A}_{k}(j):=(1 / \sqrt{ } d)\left(\hat{\tilde{H}}_{k+1}\left(j+D^{k} \ell_{1}\right)|\cdots| \hat{\tilde{H}}_{k+1}\left(j+D^{k} \ell_{d}\right)\right) .
\end{array}\right\}
$$

Then (3.7) is equivalent to

$$
A_{k}(j) \tilde{A}_{k}(j)^{*}=I_{r}
$$

By Lemma 3.2, there exist $r d \times r d$ matrices $C_{k}(j)$ and $\tilde{C}_{k}(j)$ of the form

$$
C_{k}(j)=\left(\frac{A_{k}(j)}{B_{k}(j)}\right), \quad \tilde{C}_{k}(j)=\left(\frac{\tilde{A}_{k}(j)}{\tilde{B}_{k}(j)}\right)
$$

for some $r(d-1) \times r d$ matrices $B_{k}(j)$ and $\tilde{B}_{k}(j)$ such that

$$
C_{k}(j) \tilde{C}_{k}(j)^{*}=I_{r d}
$$

Now, define $r(d-1) \times r$ matrices $\hat{G}_{k+1}\left(j+D^{k} \ell\right), \hat{\tilde{G}}_{k+1}\left(j+D^{k} \ell\right), \ell \in \mathcal{R}_{1}$, by

$$
\left.\begin{array}{l}
B_{k}(j):=(1 / \sqrt{ } d)\left(\hat{G}_{k+1}\left(j+D^{k} \ell_{1}\right)|\cdots| \hat{G}_{k+1}\left(j+D^{k} \ell_{d}\right)\right), \\
\tilde{B}_{k}(j):=(1 / \sqrt{ } d)\left(\hat{\tilde{G}}_{k+1}\left(j+D^{k} \ell_{1}\right)|\cdots| \hat{\tilde{G}}_{k+1}\left(j+D^{k} \ell_{d}\right)\right) .
\end{array}\right\}
$$

By (3.17) and (3.18), we have

$$
\left(\begin{array}{c|c|c}
A_{k}(j) \tilde{A}_{k}(j)^{*} & A_{k}(j) \tilde{B}_{k}(j)^{*} \\
\hline B_{k}(j) \tilde{A}_{k}(j)^{*} & B_{k}(j) \tilde{B}_{k}(j)^{*}
\end{array}\right)=\left(\begin{array}{c|c}
I_{r} & 0_{r \times r(d-1)} \\
\hline 0_{r(d-1) \times r} & I_{r(d-1)}
\end{array}\right),
$$

and it follows from (3.16) and (3.19) that the conditions (3.8), (3.12) and (3.13) are satisfied.

It remains to show that (3.10) holds. Note that the matrix $P_{k}(j)$ defined in (3.9) can be written as

$$
P_{k}(j)=\sum_{\ell \in \mathcal{R}_{1}} \hat{H}_{k+1}\left(j+D^{k} \ell\right) M_{k+1}\left(j+D^{k} \ell\right) \hat{G}_{k+1}\left(j+D^{k} \ell\right)^{*}
$$


Then, by (3.1) and (3.2), we see that

$$
C_{k}(j) \operatorname{diag}\left(M_{k+1}\left(j+D^{k} \ell_{1}\right), \ldots, M_{k+1}\left(j+D^{k} \ell_{d}\right)\right) C_{k}(j)^{*}=\frac{1}{d}\left(\begin{array}{c|c}
M_{k}(j) & P_{k}(j) \\
\hline P_{k}(j)^{*} & N_{k}(j)
\end{array}\right) .
$$

Since $C_{k}(j)$ is invertible and (3.3) holds, the determinant of the product of matrices in the above identity is a positive real number. Consequently, we have

$$
\operatorname{det}\left(\begin{array}{c|c}
M_{k}(j) & P_{k}(j) \\
\hline P_{k}(j)^{*} & N_{k}(j)
\end{array}\right)>0 .
$$

A similar argument shows that

$$
\operatorname{det}\left(\begin{array}{c|c}
\tilde{M}_{k}(j) & \tilde{P}_{k}(j) \\
\hline \tilde{P}_{k}(j)^{*} & \tilde{N}_{k}(j)
\end{array}\right)>0
$$

and this completes the proof of the theorem.

The proof of Theorem 3.1 is constructive, and it gives an algorithm for the construction of biorthogonal multiwavelets from a pair of biorthogonal multiresolutions.

Algorithm 3.3. For $k \geqslant 0$, suppose that $\hat{H}_{k+1}, \hat{\tilde{H}}_{k+1} \in \mathcal{S}\left(D^{k+1}\right)^{r \times r}$ are the matrices in the periodic matrix refinement equations (1.3).

Step 1. Fix $j \in \mathcal{R}_{k}$ and form the $r \times r d$ matrices $A_{k}(j)$ and $\tilde{A}_{k}(j)$ as in (3.16).

Step 2. Find a $(r d-r) \times r d$ matrix $\tilde{B}_{k}(j)$ whose rows formed a basis of the orthogonal complement of the linear span of the rows of $A_{k}(j)$ in $\mathbb{C}^{r d}$.

Step 3. Set $B_{k}(j)$ to be the $(r d-r) \times r d$ matrix formed by the last $(r d-r)$ rows of the $r d \times r d$ matrix $\left(\tilde{A}_{k}(j)^{*} \mid \tilde{B}_{k}(j)^{*}\right)^{-1}$.

Step 4. Define $r(d-1) \times r$ matrices $\hat{G}_{k+1}\left(j+D^{k} \ell\right), \tilde{\tilde{G}}_{k+1}\left(j+D^{k} \ell\right), \ell \in \mathcal{R}_{1}$, by (3.19).

Step 5. Repeat steps 1-4 with all the elements $j$ in $\mathcal{R}_{k}$.

Step 6. Write $\psi_{k}=\left(\psi_{k}^{1}, \ldots, \psi_{k}^{r(d-1)}\right)^{\mathrm{T}}$ and $\tilde{\psi}_{k}=\left(\tilde{\psi}_{k}^{1}, \ldots, \tilde{\psi}_{k}^{r(d-1)}\right)^{\mathrm{T}}$, and set

$$
\begin{aligned}
\psi_{k}(x) & :=\sum_{n \in \mathbb{Z}^{s}} \hat{G}_{k+1}(n)\left(\hat{\phi}_{k+1}^{1}(n), \ldots, \hat{\phi}_{k+1}^{r}(n)\right)^{\mathrm{T}} \mathrm{e}^{\mathrm{i} n \cdot x} \\
\tilde{\psi}_{k}(x) & :=\sum_{n \in \mathbb{Z}^{s}} \hat{\tilde{G}}_{k+1}(n)\left(\hat{\tilde{\phi}}_{k+1}^{1}(n), \ldots, \hat{\tilde{\phi}}_{k+1}^{r}(n)\right)^{\mathrm{T}} \mathrm{e}^{\mathrm{i} n \cdot x},
\end{aligned}
$$

to obtain the biorthogonal multiwavelets in $L^{2}\left([0,2 \pi)^{s}\right)$.

In the following two examples on the special case of $r=1$, different values of $d$ are considered. 
Example 3.4. Let $d=2$. Then

(a) $s=1, M=2$; and

(b) $s=2, M=\left(\begin{array}{cc}1 & 1 \\ 1 & -1\end{array}\right)$ or $\left(\begin{array}{cc}1 & -1 \\ 1 & 1\end{array}\right)$ or $\left(\begin{array}{ll}0 & 2 \\ 1 & 0\end{array}\right)$,

are some examples of the cases included under this setting. Without loss of generality, we may assume that $\mathcal{L}_{1}=\left\{0, q_{2}\right\}$ and $\mathcal{R}_{1}=\left\{0, \ell_{2}\right\}$.

For $k \geqslant 0, j \in \mathcal{R}_{k}$, define

$$
\left.\begin{array}{rl}
\hat{\tilde{G}}_{k+1}(j) & =-(1 / \sqrt{ } 2) \mathrm{e}^{-\mathrm{i} j \cdot 2 \pi M^{-(k+1)} q_{2}} \overline{\hat{H}_{k+1}\left(j+D^{k} \ell_{2}\right)} \\
\left.+D^{k} \ell_{2}\right) & =(1 / \sqrt{ } 2) \mathrm{e}^{-\mathrm{i} j \cdot 2 \pi M^{-(k+1)} q_{2}} \overline{\hat{H}_{k+1}(j)}
\end{array}\right\}
$$

As shown independently in $[\mathbf{3}]$ and $[\mathbf{1 0}], \mathrm{e}^{-\mathrm{i} \ell_{2} \cdot 2 \pi M^{-1} q_{2}}=-1$. This implies that the two equations in (3.20) can be combined together as

$$
\hat{\tilde{G}}_{k+1}(j)=-(1 / \sqrt{ } 2) \mathrm{e}^{-\mathrm{i} j \cdot 2 \pi M^{-(k+1)} q_{2}} \overline{\hat{H}_{k+1}\left(j+D^{k} \ell_{2}\right)}, \quad j \in \mathcal{R}_{k+1} .
$$

Now, for $k \geqslant 0, j \in \mathcal{R}_{k}$, the matrix $\tilde{C}_{k}(j)$ in (3.17) is given by

$$
\tilde{C}_{k}(j)=\frac{1}{\sqrt{ } 2}\left(\begin{array}{cc}
\hat{\tilde{H}}_{k+1}(j) & \hat{\tilde{H}}_{k+1}\left(j+D^{k} \ell_{2}\right) \\
-\mathrm{e}^{-\mathrm{i} j \cdot 2 \pi M^{-(k+1)} q_{2}} \frac{\hat{H}_{k+1}\left(j+D^{k} \ell_{2}\right)}{\mathrm{e}^{-\mathrm{i} j \cdot 2 \pi M^{-(k+1)} q_{2}} \frac{\hat{H}_{k+1}(j)}{{ }^{2}}}
\end{array}\right) .
$$

Then the matrix $C_{k}(j)=\left(\tilde{C}_{k}(j)^{*}\right)^{-1}$ in (3.17) yields

$$
\hat{G}_{k+1}(j)=-(1 / \sqrt{ } 2) \mathrm{e}^{-\mathrm{i} j \cdot 2 \pi M^{-(k+1)} q_{2}} \overline{\tilde{\hat{H}}}_{k+1}\left(j+D^{k} \ell_{2}\right), \quad j \in \mathcal{R}_{k+1} .
$$

Note that (3.21) and (3.22) are equivalent to

$$
\tilde{G}_{k+1}(\ell)=\mathrm{e}^{-\mathrm{i} \ell_{2} \cdot 2 \pi M^{-1} \ell} \overline{H_{k+1}\left(q_{2}-\ell\right)}, \quad G_{k+1}(\ell)=\mathrm{e}^{-\mathrm{i} \ell_{2} \cdot 2 \pi M^{-1} \ell} \overline{\tilde{H}_{k+1}\left(q_{2}-\ell\right)},
$$

for all $\ell \in \mathcal{L}_{k+1}$. In the special case of $s=1$ and $M=2$, this becomes

$$
\tilde{G}_{k+1}(\ell)=(-1)^{\ell} \overline{H_{k+1}(1-\ell)}, \quad G_{k+1}(\ell)=(-1)^{\ell} \overline{\tilde{H}_{k+1}(1-\ell)},
$$

for all $\ell=0,1, \ldots, 2^{k+1}-1$. These expressions are periodic analogues of the familiar alternating flip formulae for the construction of wavelets in $L^{2}(\mathbb{R})$.

Example 3.5. Let $d>2$, and fix $k \geqslant 0, j \in \mathcal{R}_{k}$. Without loss of generality, suppose that $\ell_{1}, \ell_{2}, \ldots, \ell_{d}$ are all the elements of $\mathcal{R}_{1}$ with

$$
\left|\hat{H}_{k+1}\left(j+D^{k} \ell_{1}\right)\right|=\max _{\ell \in \mathcal{R}_{1}}\left|\hat{H}_{k+1}\left(j+D^{k} \ell\right)\right| .
$$

Define the $(d-1) \times 1$ matrices

$$
\hat{\tilde{G}}_{k+1}\left(j+D^{k} \ell\right):=\left(\hat{\tilde{G}}_{k+1}^{1}\left(j+D^{k} \ell\right), \ldots, \hat{\tilde{G}}_{k+1}^{d-1}\left(j+D^{k} \ell\right)\right)^{\mathrm{T}}, \quad \ell \in \mathcal{R}_{1},
$$


by

$$
\hat{\tilde{G}}_{k+1}^{m}\left(j+D^{k} \ell_{\nu}\right)= \begin{cases}-(1 / \sqrt{ } d) \overline{\hat{H}_{k+1}\left(j+D^{k} \ell_{m+1}\right)}, & \text { if } \nu=1, \\ (1 / \sqrt{ } d) \overline{\hat{H}_{k+1}\left(j+D^{k} \ell_{1}\right)}, & \text { if } \nu=m+1 \\ 0, & \text { otherwise, }\end{cases}
$$

where $m=1,2, \ldots, d-1, \nu=1,2, \ldots, d$. Then the matrix $\tilde{C}_{k}(j)$ in (3.17) is given by

$$
\begin{aligned}
& \tilde{C}_{k}(j)=\frac{1}{\sqrt{ } d} \times \\
& \left(\begin{array}{ccccc}
\frac{\hat{\tilde{H}}_{k+1}\left(j+D^{k} \ell_{1}\right)}{\hat{\hat{H}}_{k+1}\left(j+D^{k} \ell_{2}\right)} & \frac{\hat{\tilde{H}}_{k+1}\left(j+D^{k} \ell_{2}\right)}{\hat{H}_{k+1}\left(j+D^{k} \ell_{1}\right)} & \hat{\tilde{H}}_{k+1}\left(j+D^{k} \ell_{3}\right) & \cdots & \hat{\tilde{H}}_{k+1}\left(j+D^{k} \ell_{d}\right) \\
-\frac{\hat{H}_{k+1}\left(j+D^{k} \ell_{3}\right)}{\hat{H}_{k+1}\left(j+D^{k} \ell_{1}\right)} & 0 & \ldots & \cdots & 0 \\
\vdots & \vdots & \ldots & 0 & 0 \\
-\hat{\hat{H}}_{k+1}\left(j+D^{k} \ell_{d}\right) & 0 & \ldots & 0 & \hat{H}_{k+1}\left(j+D^{k} \ell_{1}\right)
\end{array}\right),
\end{aligned}
$$

and a direct calculation yields

$$
\operatorname{det}\left(\tilde{C}_{k}(j)\right)=\left(\frac{1}{\sqrt{ } d}\right)^{d}{\overline{\hat{H}_{k+1}\left(j+D^{k} \ell_{1}\right)}}^{d-2} \sum_{\nu=1}^{d} \hat{\tilde{H}}_{k+1}\left(j+D^{k} \ell_{\nu}\right) \overline{\hat{H}_{k+1}\left(j+D^{k} \ell_{\nu}\right)} .
$$

Consequently, it follows from (3.7) that

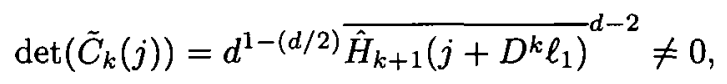

since $\left|\hat{H}_{k+1}\left(j+D^{k} \ell_{1}\right)\right|=\max _{\ell \in \mathcal{R}_{1}}\left|\hat{H}_{k+1}\left(j+D^{k} \ell\right)\right|>0$.

The $(d-1) \times 1$ matrices $\hat{G}_{k+1}\left(j+D^{k} \ell\right), \ell \in \mathcal{R}_{1}$, are obtained from the matrix $C_{k}(j)$ in (3.17), which is given by

$$
C_{k}(j)=\left(\tilde{C}_{k}(j)^{*}\right)^{-1}=\frac{1}{d^{1-(d / 2)} \hat{H}_{k+1}\left(j+D^{k} \ell_{1}\right)^{d-2}} \operatorname{adj}\left(\tilde{C}_{k}(j)^{*}\right)
$$

where $\operatorname{adj}\left(\tilde{C}_{k}(j)^{*}\right)$ denotes the transpose of the matrix of cofactors of $\tilde{C}_{k}(j)^{*}$.

\section{Biorthogonal Riesz bases}

For $k \geqslant 0$, suppose that $\left\{T_{k}^{\ell} \psi_{k}^{m}: m=1,2, \ldots, r(d-1), \ell \in \mathcal{L}_{k}\right\}$ and $\left\{T_{k}^{\ell} \tilde{\psi}_{k}^{m}: m=\right.$ $\left.1,2, \ldots, r(d-1), \ell \in \mathcal{L}_{k}\right\}$ form a pair of biorthogonal bases of $W_{k}$ and $\tilde{W}_{k}$, respectively. Since $L^{2}\left([0,2 \pi)^{s}\right)$ has the direct sum decompositions

$$
L^{2}\left([0,2 \pi)^{s}\right)=V_{0} \oplus W_{0} \oplus W_{1} \oplus \cdots=\tilde{V}_{0} \oplus \tilde{W}_{0} \oplus \tilde{W}_{1} \oplus \cdots,
$$

it is natural to ask whether the two collections of functions

$$
\left\{\phi_{0}^{m}: m=1,2, \ldots, r\right\} \cup\left\{T_{k}^{\ell} \psi_{k}^{m}: k \geqslant 0, m=1,2, \ldots, r(d-1), \ell \in \mathcal{L}_{k}\right\}
$$


and

$$
\left\{\tilde{\phi}_{0}^{m}: m=1,2, \ldots, r\right\} \cup\left\{T_{k}^{\ell} \tilde{\psi}_{k}^{m}: k \geqslant 0, m=1,2, \ldots, r(d-1), \ell \in \mathcal{L}_{k}\right\}
$$

form a pair of biorthogonal Riesz bases of $L^{2}\left([0,2 \pi)^{s}\right)$. In this connection, the following theorem, which is a multidimensional periodic analogue of results in $[\mathbf{3}, \mathbf{5}, \mathbf{6}]$, on a general sequence of functions $\psi_{k}^{m}, k \geqslant k_{0}, m=1,2, \ldots, r(d-1)$, is useful.

Theorem 4.1. Suppose that there exist positive constants $C_{1}$ and $C_{2}$ such that

$$
\sum_{k=k_{0}}^{\infty} \sum_{m=1}^{r(d-1)}\left|\sqrt{d^{k}} \hat{\psi}_{k}^{m}(n)\right|^{\epsilon} \leqslant C_{1}, \quad n \in \mathbb{Z}^{s},
$$

and

$$
\sum_{p \in \mathbb{Z}^{s}}\left|\sqrt{d^{k}} \hat{\psi}_{k}^{m}\left(j+D^{k} p\right)\right|^{2-\epsilon} \leqslant C_{2}, \quad k \geqslant k_{0}, \quad m=1,2, \ldots, r(d-1), \quad j \in \mathcal{R}_{k},
$$

for some $k_{0} \geqslant 0$ and $\epsilon>0$. Then there exists a positive constant $B_{0}$ such that, for every $f \in L^{2}\left([0,2 \pi)^{s}\right)$,

$$
\sum_{k=k_{0}}^{\infty} \sum_{m=1}^{r(d-1)} \sum_{\ell \in \mathcal{L}_{k}}\left|\left\langle f, T_{k}^{\ell} \psi_{k}^{m}\right\rangle\right|^{2} \leqslant B_{0}\|f\|^{2} .
$$

Proof. First, fix $k \geqslant k_{0}$. By Parseval's identity,

$$
\begin{aligned}
\sum_{m=1}^{r(d-1)} \sum_{\ell \in \mathcal{L}_{k}}\left|\left\langle f, T_{k}^{\ell} \psi_{k}^{m}\right\rangle\right|^{2} & =\sum_{m=1}^{r(d-1)} \sum_{\ell \in \mathcal{L}_{k}}\left|\sum_{n \in \mathbb{Z}^{s}} \hat{f}(n) \overline{\hat{\psi}_{k}^{m}(n)} \mathrm{e}^{\mathrm{i} n \cdot\left(2 \pi M^{-k} \ell\right)}\right|^{2} \\
& =\sum_{m=1}^{r(d-1)} \sum_{\ell \in \mathcal{L}_{k}}\left|\sum_{j \in \mathcal{R}_{k}} \sum_{p \in \mathbb{Z}^{s}} \hat{f}\left(j+D^{k} p\right) \overline{\hat{\psi}_{k}^{m}\left(j+D^{k} p\right)} \mathrm{e}^{\mathrm{i} j \cdot\left(2 \pi M^{-k} \ell\right)}\right|^{2} .
\end{aligned}
$$

Let

$$
\hat{\alpha}_{k}^{m}(j):=\sum_{p \in \mathbb{Z}^{s}} \hat{f}\left(j+D^{k} p\right) \overline{\hat{\psi}_{k}^{m}\left(j+D^{k} p\right)}, \quad j \in \mathcal{R}_{k} .
$$

Then $\hat{\alpha}_{k}^{m} \in \mathcal{S}\left(D^{k}\right)$, and its inverse finite Fourier transform $\alpha_{k}^{m}$ is a sequence in $\mathcal{S}\left(M^{k}\right)$. Furthermore, $\hat{\alpha}_{k}^{m}$ and $\alpha_{k}^{m}$ satisfy the relation (1.1). Consequently, it follows from (4.6) that

$$
\begin{aligned}
\sum_{m=1}^{r(d-1)} \sum_{\ell \in \mathcal{L}_{k}}\left|\left\langle f, T_{k}^{\ell} \psi_{k}^{m}\right\rangle\right|^{2} & =\sum_{m=1}^{r(d-1)} \sum_{\ell \in \mathcal{L}_{k}}\left|\sum_{j \in \mathcal{R}_{k}} \hat{\alpha}_{k}^{m}(j) \mathrm{e}^{\mathrm{i} j \cdot\left(2 \pi M^{-k} \ell\right)}\right|^{2} \\
& =\sum_{m=1}^{r(d-1)}\left|\mathcal{R}_{k}\right|^{2} \sum_{\ell \in \mathcal{L}_{k}}\left|\alpha_{k}^{m}(\ell)\right|^{2} \\
& =\sum_{m=1}^{r(d-1)}\left|\mathcal{R}_{k}\right| \sum_{j \in \mathcal{R}_{k}}\left|\hat{\alpha}_{k}^{m}(j)\right|^{2} .
\end{aligned}
$$


This implies that, for $\epsilon>0$,

$$
\begin{aligned}
& \sum_{m=1}^{r(d-1)} \sum_{\ell \in \mathcal{L}_{k}}\left|\left\langle f, T_{k}^{\ell} \psi_{k}^{m}\right\rangle\right|^{2} \\
& \leqslant d^{k} \sum_{m=1}^{r(d-1)} \sum_{j \in \mathcal{R}_{k}}\left(\sum_{p \in \mathbb{Z}^{s}}\left|\hat{f}\left(j+D^{k} p\right)\right| \cdot\left|\hat{\psi}_{k}^{m}\left(j+D^{k} p\right)\right|^{\epsilon / 2} \cdot\left|\hat{\psi}_{k}^{m}\left(j+D^{k} p\right)\right|^{1-(\epsilon / 2)}\right)^{2} \\
& \quad \leqslant d^{k} \sum_{m=1}^{r(d-1)} \sum_{j \in \mathcal{R}_{k}}\left(\sum_{p \in \mathbb{Z}^{s}}\left|\hat{f}\left(j+D^{k} p\right)\right|^{2}\left|\hat{\psi}_{k}^{m}\left(j+D^{k} p\right)\right|^{\epsilon}\right)\left(\sum_{p \in \mathbb{Z}^{s}}\left|\hat{\psi}_{k}^{m}\left(j+D^{k} p\right)\right|^{2-\epsilon}\right) \\
& \leqslant C_{2} \sum_{m=1}^{r(d-1)} \sum_{j \in \mathcal{R}_{k}} \sum_{p \in \mathbb{Z}^{s}}\left|\hat{f}\left(j+D^{k} p\right)\right|^{2}\left|\sqrt{d^{k}} \hat{\psi}_{k}^{m}\left(j+D^{k} p\right)\right|^{\epsilon},
\end{aligned}
$$

where the assumption (4.4) is used in the last inequality.

Hence,

$$
\begin{aligned}
\sum_{k=k_{0}}^{\infty} \sum_{m=1}^{r(d-1)} \sum_{\ell \in \mathcal{L}_{k}}\left|\left\langle f, T_{k}^{\ell} \psi_{k}^{m}\right\rangle\right|^{2} & \leqslant C_{2} \sum_{k=k_{0}}^{\infty} \sum_{m=1}^{r(d-1)} \sum_{n \in \mathbb{Z}^{s}}|\hat{f}(n)|^{2}\left|\sqrt{d^{k}} \hat{\psi}_{k}^{m}(n)\right|^{\epsilon} \\
& =C_{2} \sum_{n \in \mathbb{Z}^{s}}|\hat{f}(n)|^{2} \sum_{k=k_{0}}^{\infty} \sum_{m=1}^{r(d-1)}\left|\sqrt{d^{k}} \hat{\psi}_{k}^{m}(n)\right|^{\epsilon} .
\end{aligned}
$$

Then (4.5) follows from the assumption (4.3).

Theorem 4.2. For each of the collections of functions (4.1) and (4.2), suppose that there exist positive constants $C_{1}$ and $C_{2}$ such that (4.3) and (4.4) are satisfied for some $k_{0} \geqslant 0$ and $\epsilon>0$. Then (4.1) and (4.2) form a pair of biorthogonal Riesz bases of $L^{2}\left([0,2 \pi)^{s}\right)$.

Proof. By a standard argument (see [5] or [14, pp. 32-36]), it suffices to establish the existence of a positive constant $B$ such that the inequality

$$
\sum_{m=1}^{d}\left|\left\langle f, \phi_{0}^{m}\right\rangle\right|^{2}+\sum_{k=0}^{\infty} \sum_{m=1}^{r(d-1)} \sum_{\ell \in \mathcal{L}_{k}}\left|\left\langle f, T_{k}^{\ell} \psi_{k}^{m}\right\rangle\right|^{2} \leqslant B\|f\|^{2}, \quad f \in L^{2}\left([0,2 \pi)^{s}\right),
$$

holds for both collections (4.1) and (4.2). Indeed, it follows from the Cauchy-Schwarz inequality and Theorem 4.1 that, for the collection (4.1),

$$
\begin{aligned}
& \sum_{m=1}^{d}\left|\left\langle f, \phi_{0}^{m}\right\rangle\right|^{2}+\sum_{k=0}^{\infty} \sum_{m=1}^{r(d-1)} \sum_{\ell \in \mathcal{L}_{k}}\left|\left\langle f, T_{k}^{\ell} \psi_{k}^{m}\right\rangle\right|^{2} \\
& \quad \leqslant\left(\sum_{m=1}^{d}\left\|\phi_{0}^{m}\right\|^{2}+\sum_{k=0}^{k_{0}-1} \sum_{m=1}^{r(d-1)} \sum_{\ell \in \mathcal{L}_{k}}\left\|T_{k}^{\ell} \psi_{k}^{m}\right\|^{2}+B_{0}\right)\|f\|^{2},
\end{aligned}
$$


where the constant $B_{0}$ is as in (4.5). Similarly, the collection (4.2) satisfies another such inequality. Thus the proof of the theorem is complete.

For the special case of $r=1$ and $d=2$ on the space $L^{2}\left(\mathbb{R}^{s}\right)$, the compactly supported biorthogonal wavelets $\psi$ and $\tilde{\psi}$ in $[\mathbf{5}, \mathbf{6}]$ satisfy the conditions

$$
\sup _{\omega \in \mathbb{R}^{s}} \sum_{k=-\infty}^{\infty}\left|\hat{\psi}\left(D^{k} \omega\right)\right|^{\epsilon}<\infty, \quad \sup _{\omega \in \mathbb{R}^{s}} \sum_{k=-\infty}^{\infty}\left|\hat{\tilde{\psi}}\left(D^{k} \omega\right)\right|^{\tilde{\epsilon}}<\infty
$$

and

$$
\sup _{\omega \in \mathbb{R}^{s}} \sum_{p \in \mathbb{Z}^{s}}|\hat{\psi}(\omega+2 \pi p)|^{2-\epsilon}<\infty, \quad \sup _{\omega \in \mathbb{R}^{s}} \sum_{p \in \mathbb{Z}^{s}}|\hat{\tilde{\psi}}(\omega+2 \pi p)|^{2-\tilde{\epsilon}}<\infty
$$

where $\epsilon$ and $\tilde{\epsilon}$ are positive constants. For $k \geqslant 0$, let $\psi_{k}$ and $\tilde{\psi}_{k}$ be biorthgonal wavelets in $L^{2}\left([0,2 \pi)^{s}\right)$ obtained from periodizing $\psi$ and $\tilde{\psi}$ with the formulae:

$$
\psi_{k}(x)=\sqrt{d^{k}} \sum_{p \in \mathbb{Z}^{s}} \psi\left(\frac{M^{k}}{2 \pi} x+M^{k} p\right), \quad \tilde{\psi}_{k}(x)=\sqrt{d^{k}} \sum_{p \in \mathbb{Z}^{s}} \tilde{\psi}\left(\frac{M^{k}}{2 \pi} x+M^{k} p\right) .
$$

Then it follows from the conditions (4.7) and (4.8) that $\psi_{k}$ and $\tilde{\psi}_{k}$ satisfy (4.3) and (4.4) for some $k_{0}$. On a related note, suppose that $\phi_{k}$ and $\tilde{\phi}_{k}, k \geqslant 0$, are biorthogonal scaling functions in $L^{2}\left([0,2 \pi)^{s}\right)$ obtained by periodizing the compactly supported biorthogonal scaling functions of $L^{2}\left(\mathbb{R}^{s}\right)$ in $[\mathbf{5}, \mathbf{6}]$. Then the matrix extension procedure in Example 3.4 also yields the same biorthogonal wavelets $\psi_{k}$ and $\tilde{\psi}_{k}, k \geqslant 0$, which satisfy (4.3) and (4.4).

There are still many open problems in the topic of multidimensional biorthogonal periodic multiwavelets. For instance, for the space $L^{2}\left(\mathbb{R}^{s}\right)$, the study of biorthogonal scaling functions and multiwavelets can be formulated in terms of transition operators and cascade algorithms. It would be interesting to derive such an approach for the space $L^{2}\left([0,2 \pi)^{s}\right)$.

Acknowledgements. We thank Professor S. L. Lee of the National University of Singapore and Professor Rong-Qing Jia of the University of Alberta for helpful suggestions and insightful discussions.

\section{References}

1. H. L. Chen, Wavelets from trigonometric spline approach, J. Approx. Theory Appl. 12 (1996), 99-110.

2. H. L. CHEN, Construction of orthonormal wavelets in the periodic case, Chinese Sci. Bull. 14 (1996), 552-554.

3. C. K. ChUi AND C. LI, A general framework of multivariate wavelets with duals, Appl. Comput. Harmonic Analysis 1 (1994), 368-390.

4. C. K. Chui And N. H. Mhaskar, On trigonometric wavelets, Constr. Approx. 9 (1993), $167-190$.

5. A. Cohen And I. Daubechies, A stability criterion for biorthogonal wavelet bases and their related subband coding scheme, Duke Math. J. 68 (1992), 313-335. 
6. A. Cohen And I. Daubechies, Non-separable bidimensional wavelet bases, Rev. Mat. Iberoamericana 9 (1993), 51-137.

7. P. J. Davis, Circulant matrices (Wiley, 1979).

8. S. S. GOH, S. L. LEE AND K. M. TEO, Multidimensional periodic multiwavelets, $J$. Approx. Theory 98 (1999), 72-103.

9. S. S. GOH AND V. B. YAP, Matrix extension and biorthogonal multiwavelet construction, Linear Algebra Appl. 269 (1998), 139-157.

10. T. N. T. Goodman, Construction of wavelets with multiplicity, Rendiconti Matematica 15 (1994), 665-691.

11. Y. W. KOH, S. L. LeE AND H. H. TAN, Periodic orthogonal splines and wavelets, Appl. Comput. Harmonic Analysis 2 (1995), 201-218.

12. F. J. NARCOWICH AND J. D. WARD, Wavelets associated with periodic basis functions, Appl. Comput. Harmonic Analysis 3 (1996), 40-56.

13. G. Plonka AND M. TASche, On the computation of periodic spline wavelets, Appl. Comput. Harmonic Analysis 2 (1995), 1-14.

14. I. J. YounG, An introduction to nonharmonic Fourier series (Academic Press, 1980). 